\title{
EREBEA
}

Revista de Humanidades y Ciencias Sociales

Núm. 10 (2020), pp. 111-128

ISSN: 0214-0691

http://dx.doi.org/10.33776/erebea.v10i0.4958

\section{Positive Youth Development: The 5 C's EFFECT IN A SCHOOL ENVIRONMENT}

\author{
Gina Tomé $1,2,3,5$ \\ Margarida Gaspar de Matos ${ }^{1,2}$ \\ Marta Reis ${ }^{1,2,4}$ \\ Lúcia Ramiro ${ }^{1,2}$ \\ Filipa Coelhoso ${ }^{1,2,5}$ \\ Diego Gomez-Baya ${ }^{1,6}$ \\ Nora Wiium ${ }^{7}$ \\ ${ }^{1}$ Universidade de Lisboa, Faculdade de Motricidade Humana \\ (Equipa Aventura Social), Portugal \\ ${ }^{2}$ Instituto de Saúde Ambiental, Faculdade de Medicina, \\ Universidade de Lisboa, Portugal \\ ${ }^{3}$ Bolseira Pós Doutoramento FCT SFRH/BPD/108637/2015 \\ ${ }^{4}$ Bolseira Pós Doutoramento FCT SFRH/BPD/110905/2015 \\ ${ }^{5}$ Instituto Superior de Ciências Educativas, ISCE \\ ${ }^{6}$ Department of Social, Developmental and Educational Psychology, \\ Universidad de Huelva, Spain \\ ${ }^{7}$ Department of Psychosocial Science, Faculty of Psychology, \\ University of Bergen, Norway

\section{RESUMEN} \\ La perspectiva del Desarrollo Positivo \\ Juvenil (DPJ) se centra en reconocer \\ las fortalezas psicosociales y fomentar \\ los entornos sociales que contribuyan \\ al buen desarrollo del adolescente. El \\ contexto escolar es donde los adolescentes \\ pasan la mayor parte de su tiempo y \\ donde desarrollan la mayor parte de sus \\ competencias. Es importante comprender \\ cómo se pueden promover estas
}

Abstract

A positive youth development perspective focuses on recognizing psychosocial strengths and providing social environments that contribute to the adolescent's development. The school context is where adolescents spend most of their time and where they develop most of their competencies. It is important to understand how these competencies can be promoted and how they can influence 
your well-being. The study aimed was to analyse the impact of the PYD 5 $\mathrm{C}^{\prime} \mathrm{s}$ on Portuguese adolescents in their school environment. Participants were students between 5th grade and 12th grade that attend public schools. The questionnaire was administered to 384 adolescents. The results indicated the importance of 5 C's supporting to promote positive interpersonal relationships and, consequently for the adolescent's well-being. Our research revealed that adolescents feelings in school environment are essentially influenced by their own competences.

KEYWORDS

Positive Youth Development; Adolescents; School Environments, Teacher Relationships, Well-being;

Fecha de recepción: 24 de septiembre de 2020 Fecha de aceptación: 26 de octubre de 2020 competencias y cómo pueden influir en su bienestar. El estudio tuvo como objetivo analizar el impacto de las 5 Cs de DPJ en los adolescentes portugueses en su entorno escolar. Los participantes fueron estudiantes de educación primaria y secundaria que asisten a escuelas públicas. El cuestionario se administró a 384 adolescentes. Los resultados indicaron la importancia del apoyo de las 5 Cs para promover relaciones interpersonales positivas y, en consecuencia, para promocionar el bienestar del adolescente. Nuestra investigación reveló que los sentimientos de los adolescentes en el entorno escolar están primcipalmente influidos por sus propias competencias.

Palabras Clave

Desarrollo Positivo Juvenil; Adolescentes; Entorno escolar, relaciones con los profesores, bienestar.

\section{ACKNOWLedgments}

To Nora Wiium and the other colleagues from PYD international: http:// www.uib.no/en/rg/sipa/pydcrossnational for all support and inspiration, and colleagues from Aventura Social www.aventurasocial.com 


\section{INTRODUCTION}

A positive youth development perspective focuses on recognizing psychosocial strengths and providing for social environments that contribute to the adolescent's development. The PYD perspective views children and adolescents as resources to be developed rather than problems to be fixed or managed and identifies five "C's" that characterize optimal development: caring, character, competence, confidence, and connection (Richardson et al., 2017).

Geldhof et al. (2019) refer that the primary hypothesis underlying the Five C's Model is that positive youth development can be described by five broad constructs-competence, confidence, character, caring, and connection. In other words, when youngsters exhibit all five of the C's, they are likely experiencing adaptive developmental regulations. The Five C's reflect prominent terms used by practitioners, parents, and youngsters when in concept they describe the main characteristics of "thriving youth" (Geldhof et al., 2019). The positive impact that the five C's promotion have on the youth's health is notorious, as attested byseveral studies (Gomez-Baya et al., 2019; Holsen et al., 2017; Matos et al., 2017, 2018) little is known about gender differences in PYD and its outcomes in thriving and social engagement, which could improve interventionsl" effectiveness. Thus, this study aimed to examine gender differences in PYD dimensions (i.e., competence, confidence, connection, caring, and character.

Worker et al. (2019) refers that PYD promotes a holistic perspective of youth well-being and thus integrates multiple outcome indicators. As a whole, PYD framework promotes a holistic viewpoint of well-being while emphasizing the interdependent role between individual agency and supportive environments. A primary assumption is that positive development is promoted across the lifespan when the ecological setup surrounding an individual are well arranged, by oneself and/or others (Worker et al., 2019).

Research to date has consistently noted some gender differences in psychological adjustment, lifestyles, and academic adjustment during youth life period (Gomez-Baya et al., 2019). For example, Shakya and collaborators (2019) considers that some sex-specific differences in health outcomes are caused by biological factors, many others are socially driven through gender norms. In their study they found out that adolescent gender expression is correlated with health in adulthood independently of gender expression as an adult. So, those designing 
health behaviour interventions should carefully consider integrating gender transformative components in their interventions (Shakya et al., 2019). The same way, that Ardal et al. (2018) found out that in general, the Five C's were more important for female students' perception of school empowerment and school satisfaction, than that for male perception. The difference between the mediating effect of efficacious C's and socio-emotional C's on the relationship between school empowerment and school satisfaction may be explained by the fact that several items, measuring efficacious C's, are closely linked to the school context. For example, connection is measured by teacher support, while competence is measured by academic competence (Årdal et al., 2018)as well as gender differences in these relationships. The data stemmed from a cross-sectional survey of 997 adolescents from four upper secondary schools in Norway. Structural equation modelling (SEM.

So, it is assumed that environments in which adolescents are insert in are of great importance for their development. The school is a primary location for the development of personal and social skills that help adolescents to promote their health and well-being (Tomé et al. 2019). Schools have been identified as a main scenery for building social, emotional, and behavioural results because students spend a substantial amount of time there. The school also provides a socialising context in which students are able to learn a range of skills, many of which are associated with academic achievement, and it is considered an exclusive scenery within which yougsters' social and emotional well-being can be promoted and critical skills for school, work and life can be learned and practiced (Goldberg et al., 2019).

Teachers also have an essential role in the adolescent's development. According to Hattie (2009), among the factors that help students' learning is proximity and trust with teachers. If teachers do not create a close relationship with their students, they will likely not going to be able to develop their own relationships and interactions with their peers, creating also a negative feeling for the school environment and broader community.

Tomé et al. (2019) found that the relationship with teachers was the variable with the greatest effect on school satisfaction among Portuguese adolescents. On the other hand, satisfaction with school appears positively associated with life satisfaction and happiness, and negatively with social alienation, psychological symptoms and concerns. Thus the correlation becomes more obvious - adolescents who are more satisfied with school are more satisfied with life in general and happier.

This study aimed for analysing the impact of the PYD 5 C's in a school environment, among Portuguese adolescents, in order to verify its well-being influence, teachers and colleague's relationship influence and school feelings influence. 


\section{Methods}

\section{PROCEDURE}

The ES'COOL Project arises from the gap in the promotion of mental health in a school context. The main goal of ES'COOL is to promote adolescents' mental health through capacity building of school teachers and staff. The program aims developing personal and social skills. It includes the prevention of anxiety and depression symptoms and the promotion of resiliency, and self-regulation in adolescents. Teachers training will allow not only an early detection of problems that affect adolescents' mental health, but also an early intervention, which improves care effectiveness (Tomé, Matos, Camacho, \& Gomes, 2019; Tomé et al., 2018; Tomé, Matos, Gomes, Camacho, \& Gaspar, 2017).

To evaluate the teacher training impact, teachers were asked to send a link to the questionnaire to their students, at the starting date and a month after the training was complete. The questionnaire link was sent only to students who gave their concent to participate. The inclusion criterion of the study was to be a student of one of the teachers who was conducting the training.

The student's survey consists of questions about their mental health, wellbeing, school and teachers' relationships and the PYD questionnaire. Both questionnaires (teachers and adolescents) were submitted and approved by the Santa Maria Hospital Ethics Committee and informed consent was obtained from the caregivers in the case of adolescents under the age of 18 years (Tomé et al., 2019; Tomé et al., 2018; Tomé et al., 2017).

\section{PARTICIPANTS}

The sample was constituted by students from teachers who participated in the ES'COOL Training. The questionnaire was administered to 384 adolescents, $53.4 \%$ were male, aged between 10 and 20 years $(M=15.3, S D=2.3)$. Participants were 5 th to 12 th grade students attending national public schools (see table 1 ) (Tomé et al., 2019; Tomé et al., 2018; Tomé et al., 2017).

\section{INSTRUMENT}

The original version of the Positive Youth Development was developed with data from the 4-H Study (Bowers et al., 2010; Jelicic, Bobek, Phelps, Lerner, \& Lerner, 2007; Lerner et al., 2005) with samples of adolescents aged between 10 and 16 years old.

A shorter version of the Positive Youth Development Short Form (PYD-SF) was later constructed. The scale was narrowed down from 78 to 34 items and translated into Portuguese (Geldhof et al., 2014). 
More recently, Matos et al. (2018), validated a version with 20 items, destined to youngsters between ages of 16 and 29 years old, where the 34 items scale was used, with an answer option ranging from 1 (totally disagree) to 5 (totally agree).

Life satisfaction, teacher's relationship, colleague's relationship and school feeling was measured with the scale of Cantril (1965), graphically represented as a ladder, where step " 10 " corresponds to "best possible" and step "0" represents "the worst possible".

\section{STATISTICAL ANALYSIS}

Data was analysed through the statistics program SPSS 24. The statistical analysis considered most appropriate to meet the study objective and variables type used were internal, bivariate correlation analysis and multiple linear regressions.

\section{RESULTS}

The internal consistency values of the subscales used in this study are good, with ranging from $\alpha=.73$ to $\alpha=.88$ and can be found in the previous article by Tomé et al. (2020).

Findings from the correlation analysis reveal a positive and significant correlation between competence and: confidence $(r=.723, p \leq .001)$, character $(r=.437, p \leq .001)$, caring $(r=.269, p \leq .001)$, connection $(r=.641, p \leq .001)$, life satisfaction ( $r=.376, p \leq .001)$, teacher's relationships $(r=.359, p \leq .001)$, colleague's relationships $(r=.369, p \leq .001)$ and school feelings $(r=.328, p \leq .001)$; confidence and: character $(r=.446, p \leq .001)$, caring $(r=.236, p \leq .001)$, connection $(r=.631$, $p \leq .001)$, life satisfaction $(r=.488, p \leq .001)$, teacher's relationships $(r=.341$, $p \leq .001)$, colleague's relationships $(r=.354, p \leq .001)$ and school feelings $(r=.268$, $p \leq .001)$; character and: caring $(r=.571, p \leq .001)$, connection $(r=.503, p \leq .001)$, life satisfaction $(r=.346, p \leq .001)$, teachers relationships $(r=.332, p \leq .001)$, colleagues relationships $(r=.256, p \leq .001)$ and school feelings $(r=.227, p \leq .001)$; caring and: connection $(r=.368, p \leq .001)$, life satisfaction $(r=.220, p \leq .001)$, teachers relationships $(r=.232, p \leq .001)$, colleagues relationships $(r=.174, p \leq .001)$ and school feelings $(r=.220, p \leq .001)$; connection and: life satisfaction $(r=.453$, $p \leq .001)$, teachers relationships $(r=.333, p \leq .001)$, colleagues relationships $(r=.435, p \leq .001)$ and school feelings $(r=.306, p \leq .001)$; life satisfaction and: teachers relationships $(r=.503, p \leq .001)$, colleagues relationships $(r=.522, p \leq .001)$ and school feelings $(r=.370, p \leq .001)$; teachers relationships and: colleagues relationships $(r=.396, p \leq .001)$ and school feelings $(r=.539, p \leq .001)$; and finally, colleagues relationships and: school feelings $(r=.330, p \leq .001)$. 


\begin{tabular}{l|r|r|r|r|r} 
& \multicolumn{1}{|c|}{$\mathrm{N}$} & \multicolumn{1}{c|}{$\%$} & $\mathrm{M}$ & $\mathrm{SD}$ & Range. \\
\hline Sample & & & & & \\
\hline Gender & 205 & 53.4 & & & \\
\hline Boys & 179 & 46.6 & & & \\
\hline Girls & 384 & 100 & 15.3 & 2.3 & $10-20$ \\
\hline Age & & & & & \\
\hline Grade & 8 & 2.1 & & & \\
\hline $5^{\text {th }}$ grade & 23 & 10.4 & & & \\
\hline $6^{\text {th }}$ grade & 25 & 6.5 & & & \\
\hline $7^{\text {th }}$ grade & 68 & 17.7 & & & \\
\hline $8^{\text {th }}$ grade & 84 & 21.9 & & & \\
\hline $9^{\text {th }}$ grade & 68 & 17.7 & & & \\
\hline $10^{\text {th }}$ grade & 68 & 17.7 & & & \\
\hline $11^{\text {th }}$ grade & 16 & 4.2 & & & \\
\hline $12^{\text {th }}$ grade & 368 & 95.8 & & & \\
\hline Nationality & & & & & \\
\hline Portuguese & & & \\
\hline Other & & & & \\
\hline
\end{tabular}

Table 1 - Participants' Description

\begin{tabular}{|c|c|c|c|c|c|c|c|c|c|c|c|c|}
\hline & $\mathrm{n}$ & M & SD & (1) & (2) & (3) & (4) & (5) & (6) & (7) & (8) & (9) \\
\hline (1) Competence & 384 & 21.1 & 4.4 & -- & $.723^{*}$ & $.437^{*}$ & $.269^{*}$ & $.641^{*}$ & $.376^{*}$ & $.359^{*}$ & $.369^{*}$ & $.328^{*}$ \\
\hline (2) Confidence & 384 & 22.2 & 4.9 & -- & -- & $.446^{*}$ & $.236^{*}$ & $.631^{*}$ & $.488^{*}$ & $.341^{*}$ & $.354^{*}$ & $.268^{*}$ \\
\hline (3) Character & 384 & 31.2 & 4.4 & -- & -- & -- & $.571^{*}$ & $.503^{*}$ & $.346^{*}$ & $.332^{*}$ & $.256^{*}$ & $.227^{*}$ \\
\hline (4) Caring & 384 & 24.3 & 4.8 & -- & -- & -- & -- & $.368^{*}$ & $.220^{*}$ & $.232^{*}$ & $.174^{*}$ & $.220^{*}$ \\
\hline (5) Connection & 384 & 30.4 & 5.2 & -- & -- & -- & -- & -- & $.453^{*}$ & $.333^{*}$ & $.435^{*}$ & $.306^{*}$ \\
\hline $\begin{array}{l}\text { (6)Life } \\
\text { satisfaction }\end{array}$ & 371 & 6.9 & 2 & -- & -- & -- & -- & -- & -- & $.503^{*}$ & $.522^{*}$ & $.370^{*}$ \\
\hline $\begin{array}{l}\text { (7) Teachers } \\
\text { relationship }\end{array}$ & 370 & 7.6 & 1.8 & -- & -- & -- & -- & -- & -- & -- & $.396^{*}$ & $.539^{*}$ \\
\hline $\begin{array}{l}\text { (8) Colleagues } \\
\text { relationship }\end{array}$ & 369 & 7.8 & 2.1 & -- & -- & -- & -- & -- & -- & -- & -- & $.330^{*}$ \\
\hline $\begin{array}{l}\text { (9) School } \\
\text { relationships }\end{array}$ & 369 & 6.5 & 2.7 & -- & -- & -- & -- & -- & -- & -- & -- & - \\
\hline
\end{tabular}

Table 2 - Correlations between PYD scales and environments

${ }^{*} \mathrm{p}<.001$ 
To understand the predictive effect of the Five C'S on the variables related to the adolescent's environments, multiple linear regression analysis were conducted separatly for gender and grade.

In the general model, the regression equation for the Life Satisfaction explained $27 \%$ of the variance $(R 2=.274)$. According to this model, adolescent's life satisfaction is best explained by confidence $(\beta=.349)$ and connection $(\beta=.221)$. For the Teacher Relationships variance explained $17 \% \quad(R 2=.167) \quad$ of the model. In this model, adolescent-teacher relationships are best explained by competence $(\beta=.157)$ and character $(\beta=.161)$. For the Colleagues Relationships the variance explained $19 \%(R 2=.195)$ of the model. In this model, adolescents Colleagues Relationships is best explained by connection $(\beta=.310)$. Finally, for the School Feelings variance explained $12 \%(R 2=.195)$ of the model. In this model, adolescents school feelings are best explained by competence $(\beta=.310)$.

In the boys' model, the regression equation for the Life Satisfaction explained 21\% of the variance $(R 2=.209)$. According to this model, adolescent boy's life satisfaction is best explained by confidence $(\beta=.487)$. For the boys Teacher Relationships variance explained $18 \%(R 2=.178)$ of the model. In this model, boy's teacher relationships is the best explained by confidence $(\beta=.254)$. For the Colleagues Relationships the variance explained $8 \%(R 2=.087)$ of the model. In this model, boys Colleagues Relationships is the best explained by connection $(\beta=.200)$. Finally, for the School Feelings variance explained $6 \%(R 2=.061)$ of the model. This model is not adjusted.

In the girls' model, the regression equation for the Life Satisfaction explained $41 \%$ of the variance $(R 2=.413)$. According to this model, adolescent girls life satisfaction is best explained by confidence $(\beta=.261)$ and connection $(\beta=.452)$. For the Teacher Relationships variance explained $19 \%(R 2=.191)$ of the model. In this model, girl-teacher relationships are best explained by competence $(\beta=.226)$. For the Colleagues Relationships the variance explained 31\% $(R 2=.311)$ of the model. In this model, girls Colleagues Relationships is best explained by competence $(\beta=.208)$ and connection $(\beta=.383)$. Finally, for the School Feelings variance explained $19 \%(R 2=.194)$ of the model. In this model, girls School Feelings are best explained by competence $(\beta=.228)$ and connection $(\beta=.258)$.

In the model of young people attending up to the 9th grade, the regression equation for the Life Satisfaction explained $34 \%$ of the variance $(R 2=.344)$. According to this model, youngest life satisfaction is best explained by confidence $(\beta=.415)$ and connection $(\beta=.218)$. For the Teacher Relationships variance explained $23 \%(R 2=.228)$ of the model. In this model, youngest-teacher relationships is the best explained by competence $(\beta=.303)$ and character $(\beta=.188)$. For the Colleagues Relationships the variance explained $24 \%(R 2=.239)$ of the model. In this model, youngest Colleagues Relationships is best explained by connection $(\beta=.462)$. Finally, for the School Feelings variance explained 19\% $(R 2=.191)$ of the model. In this model, youngest School Feelings are the best explained by caring $(\beta=.313)$. 


\begin{tabular}{|c|c|c|c|c|c|c|}
\hline \multirow{6}{*}{ Life Satisfaction } & Variable included & $\beta$ & $\mathrm{t}$ & $\mathrm{p}$ & $\mathrm{R}_{\mathrm{a}}^{2}$ & $\mathrm{~F}(\text { model fit })^{*}$ \\
\hline & Competence & -.068 & -.986 & .325 & \multirow{4}{*}{.274} & \multirow{4}{*}{28.872} \\
\hline & Confidence & .349 & 5.134 & .000 & & \\
\hline & Character & .100 & 1.673 & .095 & & \\
\hline & Caring & .019 & .345 & .730 & & \\
\hline & Connection & .221 & 3.457 & .001 & & \\
\hline \multirow{5}{*}{$\begin{array}{l}\text { Teachers } \\
\text { relationship }\end{array}$} & Variable included & $\beta$ & $\mathrm{t}$ & $\mathrm{p}$ & $\mathrm{R}^{2}{ }_{\mathrm{a}}$ & $\mathrm{F}(\text { model fit })^{*}$ \\
\hline & Competence & .157 & 2.128 & .034 & \multirow{4}{*}{.167} & \multirow{4}{*}{15.746} \\
\hline & Confidence & .102 & 1.399 & .163 & & \\
\hline & Character & .161 & 2.360 & .019 & & \\
\hline & Caring & .053 & .908 & .364 & & \\
\hline & Connection & .072 & 1.056 & .291 & & \\
\hline \multirow{6}{*}{$\begin{array}{l}\text { Colleagues } \\
\text { relationship }\end{array}$} & Variable included & $\beta$ & $\mathrm{t}$ & $\mathrm{p}$ & $\mathrm{R}_{\mathrm{a}}^{2}$ & $\mathrm{~F}(\text { model fit })^{*}$ \\
\hline & Competence & .106 & 1.448 & .148 & \multirow{4}{*}{.195} & \multirow{4}{*}{18.793} \\
\hline & Confidence & .073 & 1.022 & .307 & & \\
\hline & Character & .023 & .362 & .717 & & \\
\hline & Caring & .000 & -.004 & .997 & & \\
\hline & Connection & .310 & 4.597 & .000 & & \\
\hline \multirow{6}{*}{ School feelings } & Variable included & $\beta$ & $\mathrm{t}$ & $\mathrm{p}$ & $\mathrm{R}_{\mathrm{a}}^{2}$ & $\mathrm{~F}$ (model fit)* \\
\hline & Competence & .206 & 2.702 & .007 & \multirow{4}{*}{.122} & \multirow{4}{*}{11.228} \\
\hline & Confidence & .014 & .187 & .852 & & \\
\hline & Character & .006 & .092 & .926 & & \\
\hline & Caring & .112 & 1.861 & .064 & & \\
\hline & Connection & .120 & 1.705 & .089 & & \\
\hline
\end{tabular}

Table 3 - Linear Regression - Environments 
G. Tomé $\cdot$ M. Gaspar $\cdot$ M. Reis $\cdot$ L. Ramiro $\cdot$ F. Coelhoso $\cdot$ D. Gomez-Baya $\cdot$ N. Wiium

\begin{tabular}{|c|c|c|c|c|c|c|}
\hline \multirow{6}{*}{ Life Satisfaction } & Variable included & $\beta$ & $\mathrm{t}$ & $\mathrm{p}$ & $\mathrm{R}_{\mathrm{a}}^{2}$ & $\mathrm{~F}$ (model fit)* \\
\hline & Competence & -.073 & -.723 & .471 & \multirow{5}{*}{.209} & \multirow{5}{*}{11.172} \\
\hline & Confidence & .487 & 4.738 & .000 & & \\
\hline & Character & .124 & 1.314 & .190 & & \\
\hline & Caring & -.037 & -.492 & .623 & & \\
\hline & Connection & -.041 & -.433 & .665 & & \\
\hline \multirow{6}{*}{$\begin{array}{l}\text { Teachers } \\
\text { relationship }\end{array}$} & Variable included & $\beta$ & $\mathrm{t}$ & $\mathrm{p}$ & $\mathrm{R}_{\mathrm{a}}^{2}$ & $\mathrm{~F}(\text { model fit })^{*}$ \\
\hline & Competence & .149 & 1.455 & .147 & \multirow{4}{*}{.178} & \multirow{4}{*}{9.336} \\
\hline & Confidence & .254 & 2.408 & .017 & & \\
\hline & Character & .101 & 1.052 & .294 & & \\
\hline & Caring & .047 & .613 & .541 & & \\
\hline & Connection & -.032 & -.333 & .740 & & \\
\hline \multirow{6}{*}{$\begin{array}{l}\text { Colleagues } \\
\text { relationship }\end{array}$} & Variable included & $\beta$ & $\mathrm{t}$ & $\mathrm{p}$ & $\mathrm{R}_{\mathrm{a}}^{2}$ & $\mathrm{~F}$ (model fit)* \\
\hline & Competence & .004 & .032 & .974 & \multirow{4}{*}{.087} & \multirow{4}{*}{4.622} \\
\hline & Confidence & .113 & 1.018 & .310 & & \\
\hline & Character & .104 & 1.027 & .306 & & \\
\hline & Caring & -.091 & -1.105 & .271 & & \\
\hline & Connection & .200 & 1.989 & .048 & & \\
\hline \multirow{6}{*}{ School feelings } & Variable included & $\beta$ & $\mathrm{t}$ & $\mathrm{p}$ & $\mathrm{R}_{\mathrm{a}}^{2}$ & $\mathrm{~F}(\text { model fit })^{*}$ \\
\hline & Competence & .188 & 1.701 & .091 & \multirow{4}{*}{.061} & \multirow{4}{*}{3.477} \\
\hline & Confidence & .049 & .436 & .663 & & \\
\hline & Character & .044 & .430 & .668 & & \\
\hline & Caring & .107 & 1.280 & .202 & & \\
\hline & Connection & -.032 & -.315 & .753 & & \\
\hline
\end{tabular}

Table 4 - Linear Regression - Environments - Boys 


\begin{tabular}{|c|c|c|c|c|c|c|}
\hline \multirow{6}{*}{$\begin{array}{c}\text { Life } \\
\text { Satisfaction }\end{array}$} & Variable included & $\beta$ & $\mathrm{t}$ & $\mathrm{p}$ & $\mathrm{R}^{2}{ }_{\mathrm{a}}$ & $\mathrm{F}$ (model fit) ${ }^{*}$ \\
\hline & Competence & -.072 & -.843 & .400 & \multirow{4}{*}{.413} & \multirow{4}{*}{25.756} \\
\hline & Confidence & .261 & 3.132 & .002 & & \\
\hline & Character & .054 & .692 & .490 & & \\
\hline & Caring & .089 & 1.194 & .234 & & \\
\hline & Connection & .452 & 5.679 & .000 & & \\
\hline \multirow{6}{*}{$\begin{array}{l}\text { Teachers } \\
\text { relationship }\end{array}$} & Variable included & $\beta$ & $\mathrm{t}$ & $\mathrm{p}$ & $\mathrm{R}_{\mathrm{a}}^{2}$ & $\mathrm{~F}(\text { model fit })^{*}$ \\
\hline & Competence & .226 & 2.247 & .026 & \multirow{4}{*}{.191} & \multirow{4}{*}{9.332} \\
\hline & Confidence & .029 & .297 & .767 & & \\
\hline & Character & .117 & 1.286 & .200 & & \\
\hline & Caring & .041 & .464 & .643 & & \\
\hline & Connection & .168 & 1.802 & .073 & & \\
\hline \multirow{6}{*}{$\begin{array}{l}\text { Colleagues } \\
\text { relationship }\end{array}$} & Variable included & $\beta$ & $\mathrm{t}$ & $\mathrm{p}$ & $\mathrm{R}_{\mathrm{a}}^{2}$ & $\mathrm{~F}(\text { model fit })^{*}$ \\
\hline & Competence & .208 & 2.239 & .026 & \multirow{4}{*}{.311} & \multirow{4}{*}{16.855} \\
\hline & Confidence & .025 & .280 & .780 & & \\
\hline & Character & -.015 & -.178 & .859 & & \\
\hline & Caring & .064 & .788 & .432 & & \\
\hline & Connection & .383 & 4.438 & .000 & & \\
\hline \multirow{6}{*}{$\begin{array}{l}\text { School } \\
\text { feelings }\end{array}$} & Variable included & $\beta$ & $\mathrm{t}$ & $\mathrm{p}$ & $\mathrm{R}_{\mathrm{a}}^{2}$ & $\mathrm{~F}(\text { model fit })^{*}$ \\
\hline & Competence & .228 & 2.274 & .024 & \multirow{4}{*}{.194} & \multirow{4}{*}{9.456} \\
\hline & Confidence & -.019 & -.195 & .846 & & \\
\hline & Character & -.019 & -.209 & .834 & & \\
\hline & Caring & .104 & 1.194 & .234 & & \\
\hline & Connection & .258 & 2.769 & .006 & & \\
\hline
\end{tabular}

Table 5 - Linear Regression - Environments - Girls 
G. Tomé $\cdot$ M. Gaspar $\cdot$ M. Reis $\cdot$ L. Ramiro $\cdot$ F. Coelhoso $\cdot$ D. Gomez-Baya $\cdot$ N. Wilum

\begin{tabular}{|c|c|c|c|c|c|c|}
\hline \multirow{6}{*}{ Life Satisfaction } & Variable included & $\beta$ & $\mathrm{t}$ & $\mathrm{p}$ & $\mathrm{R}^{2}{ }_{\mathrm{a}}$ & $\mathrm{F}(\text { model fit })^{*}$ \\
\hline & Competence & -.083 & -.843 & .401 & \multirow{4}{*}{.344} & \multirow{4}{*}{17.674} \\
\hline & Confidence & .415 & 4.320 & .000 & & \\
\hline & Character & .152 & 1.782 & .077 & & \\
\hline & Caring & .027 & .340 & .734 & & \\
\hline & Connection & .218 & 2.448 & .016 & & \\
\hline \multirow{6}{*}{$\begin{array}{l}\text { Teachers } \\
\text { relationship }\end{array}$} & Variable included & $\beta$ & $\mathrm{t}$ & $\mathrm{p}$ & $\mathrm{R}_{\mathrm{a}}^{2}$ & $\mathrm{~F}(\text { model fit })^{*}$ \\
\hline & Competence & .303 & 2.817 & .005 & \multirow{4}{*}{.228} & \multirow{4}{*}{10.357} \\
\hline & Confidence & .026 & .251 & .802 & & \\
\hline & Character & .188 & 2.022 & .045 & & \\
\hline & Caring & -.030 & -.349 & .728 & & \\
\hline & Connection & .120 & 1.235 & .219 & & \\
\hline \multirow{6}{*}{$\begin{array}{l}\text { Colleagues } \\
\text { relationship }\end{array}$} & Variable included & $\beta$ & $\mathrm{t}$ & $\mathrm{p}$ & $\mathrm{R}_{\mathrm{a}}^{2}$ & $\mathrm{~F}(\text { model fit })^{*}$ \\
\hline & Competence & .034 & .310 & .757 & \multirow{4}{*}{.239} & \multirow{4}{*}{10.843} \\
\hline & Confidence & .080 & .768 & .444 & & \\
\hline & Character & -.063 & -.687 & .493 & & \\
\hline & Caring & .022 & .255 & .799 & & \\
\hline & Connection & .462 & 4.814 & .000 & & \\
\hline \multirow{6}{*}{$\begin{array}{l}\text { School } \\
\text { feelings }\end{array}$} & Variable included & $\beta$ & $\mathrm{t}$ & $\mathrm{p}$ & $\mathrm{R}_{\mathrm{a}}^{2}$ & $\mathrm{~F}(\text { model fit })^{*}$ \\
\hline & Competence & .106 & .951 & .343 & \multirow{4}{*}{.191} & \multirow{4}{*}{8.392} \\
\hline & Confidence & .101 & .944 & .347 & & \\
\hline & Character & -.082 & -.866 & .388 & & \\
\hline & Caring & .313 & 3.531 & .001 & & \\
\hline & Connection & .157 & 1.588 & .114 & & \\
\hline
\end{tabular}

Table 6 - Linear Regression - Environments - up to 9th grade 


\begin{tabular}{|c|c|c|c|c|c|c|}
\hline \multirow{6}{*}{$\begin{array}{c}\text { Life } \\
\text { Satisfaction }\end{array}$} & Variable included & $\beta$ & $\mathrm{t}$ & $\mathrm{p}$ & $\mathrm{R}^{2}$ & $\mathrm{~F}($ model fit)* \\
\hline & Competence & -.068 & -.701 & .484 & \multirow{4}{*}{.174} & \multirow{4}{*}{9.818} \\
\hline & Confidence & .313 & 3.236 & .001 & & \\
\hline & Character & .025 & .293 & .770 & & \\
\hline & Caring & .002 & .024 & .981 & & \\
\hline & Connection & .208 & 2.287 & .023 & & \\
\hline \multirow{6}{*}{$\begin{array}{l}\text { Teachers } \\
\text { relationship }\end{array}$} & Variable included & $\beta$ & $\mathrm{t}$ & $\mathrm{p}$ & $\mathrm{R}_{\mathrm{a}}^{2}$ & $\mathrm{~F}(\text { model fit })^{*}$ \\
\hline & Competence & .058 & .566 & .572 & \multirow{4}{*}{.093} & \multirow{4}{*}{5.322} \\
\hline & Confidence & .142 & 1.400 & .163 & & \\
\hline & Character & .125 & 1.411 & .160 & & \\
\hline & Caring & .086 & 1.084 & .280 & & \\
\hline & Connection & .027 & .289 & .773 & & \\
\hline \multirow{6}{*}{$\begin{array}{l}\text { Colleagues } \\
\text { relationship }\end{array}$} & Variable included & $\beta$ & $\mathrm{t}$ & $\mathrm{p}$ & $\mathrm{R}_{\mathrm{a}}^{2}$ & $\mathrm{~F}(\text { model fit })^{*}$ \\
\hline & Competence & .133 & 1.329 & .185 & \multirow{4}{*}{.131} & \multirow{4}{*}{7.310} \\
\hline & Confidence & .085 & .852 & .395 & & \\
\hline & Character & .056 & .641 & .522 & & \\
\hline & Caring & -.019 & -.245 & .807 & & \\
\hline & Connection & .192 & 2.059 & .041 & & \\
\hline \multirow{6}{*}{$\begin{array}{l}\text { School } \\
\text { feelings }\end{array}$} & Variable included & $\beta$ & $\mathrm{t}$ & $\mathrm{p}$ & $\mathrm{R}^{2}{ }_{\mathrm{a}}$ & $\mathrm{F}(\text { model fit })^{*}$ \\
\hline & Competence & .240 & 2.330 & .021 & \multirow{4}{*}{.075} & \multirow{4}{*}{4.403} \\
\hline & Confidence & -.030 & -.289 & .773 & & \\
\hline & Character & .043 & .484 & .629 & & \\
\hline & Caring & -.029 & -.360 & .719 & & \\
\hline & Connection & .104 & 1.081 & .281 & & \\
\hline
\end{tabular}

Table 7 - Linear Regression - Environments - 10th and 12th grade 
In the model of young people who attend school between grades 10th and 12th, the regression equation for the Life Satisfaction explained $17 \%$ of the variance $(R 2=.174)$. According to this model, older youth life satisfaction is best explained by confidence $(\beta=.313)$ and connection $(\beta=.208)$. For the Teacher Relationships variance explained $9 \%(R 2=.093)$ of the model. This model is not adjusted. For the Colleagues Relationships the variance explained $13 \%(R 2=.131)$ of the model. In this model, older youth Colleagues Relationships is the best explained by connection $(\beta=.192)$. Finally, for the School Feelings variance explained $7 \%$ $(R 2=.075)$ of the model. In this model, older youth School Feelings are the best explained by competence $(\beta=.240)$.

\section{Discussion}

The PYD perspective views children and adolescents as resources to be developed rather than problems to be fixed or managed and identifies five "C's" that characterize optimal development (Richardson et al., 2017). In this study, the 5 C's influence on the school context and Portuguese adolescents well-being was analyzed, specifically the teacher's relationship, the colleague's relationship, the school feelings and life satisfaction.

The school is the environment where adolescents spend most of their time and, consequently, the space where they keep the essential interpersonal relationships for their development and well-being. Being life satisfied can positively influence all interpersonal relationships, inside and outside the school, promoting the personal and social competences development (Tomé et al., 2019).

The results reveal the importance of the 5 C's supporting to promote positive interpersonal relationships and, consequently, for the adolescents' well-being. It was found that school feelings are essentially influenced by the adolescents' competences. When the general model is split by gender and age, the competence presence is verified in the other models, except in the younger model, in which the "caring" seems to have a greater influence than the competence, or in girls, in that the competence influence is shared with the connection.

Competence also plays an important role in the teacher's relationship with, however for boys more important than competence seems to be confidence. On the other hand, the connection emerges as essential for the colleague's relationship and the confidence and connection essential for the life satisfaction feelings, among adolescents in all models.

In the same line of these results, Worker et al. (2019) study refers that PYD promotes a holistic perspective of youth well-being. In other words, the $5 \mathrm{C}$ 's influence all analyzed environments, with different associations but significantly in all. The 5 C's promotion will allow the adolescents well-being development, consequently influencing their environments. The school is a primary location 
for the development of personal and social skills that help adolescents to promote their health and well-being (Tomé et al., 2019).

Regarding the genders difference, research has consistently noted some gender differences in psychological adjustment, lifestyles, and academic adjustment during the life stage of youth (Gomez-Baya et al., 2019; Årdal et al., 2018). The adolescents well-being promoting sometimes requires genders and age different strategies, however, the general model presented differs minimally when divided by gender and age. These differences have to be considered, without losing the essential objective of the 5 C's promoting for positive development and considering that the positive impact that the $5 \mathrm{C}$ 's promotion have on the youth health is notorious, as evidenced in several studies (Gomez-Baya et al., 2019; Holsen et al., 2017; Matos et al., 2017, 2018).

The school context is where adolescents spend most of their time and where they develop most of their competencies. It is important to understand how these competencies can be promoted and how they can influence your well-being.

\section{REFERENCES}

Årdal, E., Holsen, I., Diseth, Å., \& Larsen, T. (2018). The Five C's of Positive Youth Development in a school context; gender and mediator effects. School Psychology International, 39(1), 3-21. https://doi.org/10.1177/0143034317734416

Bowers, E. P., Li, Y., Kiely, M. K., Brittian, A., Lerner, J. V., \& Lerner, R. M. (2010). The Five C's model of positive youth development: a longitudinal analysis of confirmatory factor structure and measurement invariance. J Youth and Adolescence, 39(7), 720-735. doi:10.1007/s10964-010-9530-9.

Cantril, H. (1965) The pattern of human concerns. Rutgers University Press, New Brunswick.

Geldhof, J., Bowers, E., Boyd, M., Mueller, M., Napolitano, C., Schmid, K., Lerner, R. (2014). Creation of short and very short measures of the five C's of positive youth development. Journal of Research on Adolescence, 24(1), 163176. doi: $10.1111 /$ jora. 12039

Geldhof, G. J., Larsen, T., Urke, H., Holsen, I., Lewis, H., \& Tyler, C. P. (2019). Indicators of positive youth development can be maladaptive: The example case of caring. Journal of Adolescence, 71(November 2018), 1-9. https://doi. org/10.1016/j.adolescence.2018.11.008

Gomez-Baya, D., Reis, M., \& Gaspar de Matos, M. (2019). Positive youth development, thriving and social engagement: An analysis of gender differences in Spanish youth. Scandinavian Journal of Psychology, 60(6), 559-568. https:// doi.org/10.1111/sjop.12577 
Hattie, J. (2009). Visible learning: A synthesis of over 800 meta-analyses relating to achieve-ment. London: Routledge.

Holsen, I., Geldhof, J., Larsen, T., \& Aardal, E. (2017). The five Cs of positive youth development in Norway. International Journal of Behavioral Development, 41(5), 559-569. https://doi.org/10.1177/0165025416645668

Jelicic, H., Bobek, D. L., Phelps, E., Lerner, R. M., \& Lerner, J. V. (2007). Using positive youth development to predict contribution and risk behaviors in early adolescence: Findings from the first two waves of the 4-H study of positive youth development. International Journal of Behavioral Development, 31(3), 263-273. doi:10.1177/0165025407076439

Lerner, R. M., Lerner, J. V., Almerigi, J. B., Theokas, C., Phelps, E., Gestsdottir, S., von Eye, A. (2005). Positive Youth Development, participation in community youth development programs, and community contributions of fifth-grade adolescents: Findings from the first wave of the 4- $\mathrm{H}$ study of positive youth development. Journal of Early Adolescence, 25(1), 17-71. doi:10.1177/0272431604272461

Matos, M. G., Santos, T., \& Reis, M. (2017). BePositive project: Positive Youth Development (PYD) in adolescents. Portuguese report. L. A. Publishing Ed. Saarbrucken.

Matos, M. G., Santos, T., Reis, M., Gomez-Baya, D., \& Marques, A. (2018). Positive Youth Development in Portugal: A Tool towards Gender Equity? ARC Journal of Pediatrics, 4(1), 25-35.

Richardson, R. A., Ferguson, P. A., \& Maxymiv, S. (2017). Applying a Positive Youth Development Perspective to Observation of Bereavement Camps for Children and Adolescents. Journal of Social Work in End-of-Life \& Palliative Care, 13(2-3), 173-192. https://doi.org/10.1080/15524256.2017.1346544

Shakya, H. B., Domingue, B., Nagata, J. M., Cislaghi, B., Weber, A., \& Darmstadt, G. L. (2019). Adolescent gender norms and adult health outcomes in the USA: a prospective cohort study. The Lancet Child and Adolescent Health, 3(8), 529-538. https://doi.org/10.1016/S2352-4642(19)30160-9

Tomé, G., Matos, M.G., Reis, M., Gomez-Baya, D., Cerqueira, A., \& Wiium, N. (2020). Positive Youth Development and Mental Health among Portuguese Adolescents. European Review of Applied Psychology (submitted)

Tomé, G., Matos, M. G., Camacho, I., \& Gomes, P. (2019). Promoting Wellbeing and Mental Health in Adolescents. Cientific Journal of Neurology, 1(1), 2-7.

Tomé, G., Matos, M. G., Camacho, I., Gomes, P., Reis, M., \& Branquinho, C. (2018). Mental health promotion in school context - Validation of the ES'COOL scale for teachers. Journal of Psychiatry and Behavioral Sciences, 2, 1009. doi: http://doi.org/10.33582/2637-8027/1009. 
Tomé, G., Matos, M. G., Gomes, P., Camacho, I., \& Gaspar, T. (2017). Promoção da Saúde Mental nas Escolas - Projeto ES'COOL. Journal of Child and Adolescent Psychology, 8(1), 173-184.

Worker, S. M., Iaccopucci, A. M., Bird, M., \& Horowitz, M. (2019). Promoting Positive Youth Development Through Teenagers-as-Teachers Programs. Journal of Adolescent Research, 34(1), 30-54. https://doi. org/10.1177/0743558418764089. 
